\title{
LITERATURWISSENSCHAFT
}

http://dx.doi.org/10.18778/2196-8403.2015.15

MARION BRANDT

\section{Abschied von der Republik. Kommentare zur Preu- ßischwerdung Danzigs in der deutschsprachigen Literatur um 1800}

Artykuł analizuje, w jaki sposób współcześni pisarze komentowali zajęcie Gdańska przez Prusy w 1793 roku. Upadek Wolnego Miasta oraz negatywny stosunek obywateli Gdańska do pruskiego rządu inspirowały kilku autorów do rozważań o idealnym państwie. Anonimowy autor Briefe über Danzig (1794), Carl B. Feyerabend (Kosmopolitische Wanderungen ..., 1798) oraz August Graf von Lehndorff (Meine Reise ins blaue Ländchen ..., 1799) uważają, że absolutyzm oświeceniowy państwa pruskiego jest korzystniejszym systemem dla obywateli niż rządy republikańskie. Natomiast Johannes Daniel Falk w swej powieści autobiograficznej Leben, wunderbare Reisen und Irrfahrten des Johannes von der Ostsee (1805) ceni republikanizm wyżej niż pruski porządek. Prezentowane opinie można odczytać jako część dyskusji o idealnym państwie, jaka prowadzona była w kontekście wydarzeń rewolucji francuskiej.

Der vorliegende Aufsatz untersucht, in welcher Weise zeitgenössische Autoren die Einnahme Danzigs durch Preußen im Jahr 1793 kommentieren. Angesichts des Untergangs der Freien Stadt und einer für die Autoren signifikanten Ablehnung der preußischen Regierungsform unter den Danziger Bürgern wird die Reflexion über die ideale Staatsform zu einem der Schwerpunkte ihrer Werke. Während der anonyme Autor der Briefe über Danzig (1794), Carl B. Feyerabend (Kosmopolitische Wanderungen ..., 1798) und August Graf von Lehndorff (Meine Reise ins blaue Ländchen ..., 1799) der republikanischen Regierung den aufgeklärten Absolutismus des preußischen Staates vorziehen, verteidigt Johannes Daniel Falk in seinem autobiographischen Roman Leben, wunderbare Reisen und Irfahrten des Johannes von der Ostsee (1805) den Republikanismus gegenüber der preußischen Ordnung. Die im Aufsatz vorgestellten Positionen lassen sich als Teil einer Diskussion um die ideale Regierungsform verstehen, die im Umfeld der Französischen Revolution geführt wurde. 
The present article analyses how contemporary authors comment on the Prussian capture of Danzig in 1793. In view of the break-up of the Free City and a rejection of the Prussian government by the citizens of Danzig significant for the authors, the reflection about the ideal form of government becomes an inspiration of their texts. While the anonymous author of Briefe über Danzig (1794), Carl B. Feyerabend (Kosmopolitische Wanderungen ..., 1798) and August Graf von Lehndorff (Meine Reise ins blaue Ländchen ..., 1799) prefer the enlightened absolutism of the Prussian state to the republican government, Johannes Daniel Falk defends republicanism against the Prussian order in his autobiographical novel Leben, wunderbare Reisen und Irrfahrten des Johannes von der Ostsee (1805). The presented opinions can be regarded as a part of a discussion about the ideal form of government, which took place in the context of the French Revolution.

Mit der Besetzung der zur Polnisch-Litauischen Republik gehörenden Freien Stadt Danzig durch die preußische Armee endete im Jahr 1793 eine Epoche, in der Danzig zu den bedeutendsten europäischen Handelsstädten gehört und über politische Selbstständigkeit verfügt hatte. Es verwundert also nicht, dass der Untergang der Freistadt auf das Interesse zeitgenössischer Autoren stieß. Bei der folgenden Lektüre einiger ausgewählter Texte interessiert mich nicht die Frage, wie bzw. was sie von der Einnahme Danzigs durch Preußen erzählen, sondern vielmehr, in welcher Weise sie die neue Situation, in der sich die Stadt nach der Preußischwerdung befand, kommentieren. Zunächst wende ich mich einigen Reiseberichten vom Ende des 18. Jahrhunderts zu, um danach den 1805 veröffentlichten Roman Leben, wunderbare Reisen und Irrfahrten des Johannes von der Ostsee von Johannes Daniel Falk zu betrachten.

\section{Ausgewählte Reiseberichte}

In der Reiseliteratur vom Ende des 18. Jahrhunderts tritt Danzig selten als das Ziel einer Reise auf, sondern ist meistens eine Zwischenstation auf dem Weg aus dem Westen, oft aus Berlin, nach Königsberg oder in umgekehrter Richtung (vgl. GOLEC 1998:78, SAMP 1991:64f.). In den Berichten werden die natürliche Lage der Stadt, ihre Architektur, bekannte Kunstwerke sowie das gesellschaftliche und kaufmännische Leben nach allen Regeln der ,Reisekunst' bzw. der Apodemik beschrieben (JÄGER 1998). Drei der bekannteren Texte diskutieren darüber hinaus auch die Veränderungen des politischen Status von Danzig nach der Einnahme durch Preußen.

Die 1794 in Berlin anonym erschienenen Briefe über Danzig werden in dieser Untersuchung ähnlich wie in anderen Publikationen zur Reiseliteratur über 
Danzig ebenfalls berücksichtigt (GOLEC 1998:83-85, SAMP 1991:57) ${ }^{1}$, stellen aber im eigentlichen Sinne keinen Reisebericht dar. Der bis heute nicht ermittelte Verfasser bezeichnet sich in der Vorrede als einen „guten und redlich gesinnten Bürger dieser Stadt“ (ANONYM 1794:Vorrede, o.S.). Seine Ausführungen verraten ein reiches Insiderwissen bis hin zur Finanzlage einzelner städtischer Einrichtungen, auch schreibt er oft „uns“, wenn er von den Danziger Bürgern spricht, und von „unserer Stadt“, wenn er Danzig meint (ANONYM 1794:53).

Möglicherweise wollte der Verfasser wegen seiner Preußenfreundlichkeit anonym bleiben, denn das Anliegen seiner Schrift ist es, die Danziger Bürger von der neuen preußischen Regierung zu überzeugen und „die blinde Anhänglichkeit der Danziger an ihre alte Regierungsform - die ihnen jetzt mehr schädlich als nützlich ist - zu schwächen“ (ANONYM 1794:Vorrede,o.S.). Er beschreibt u.a. den „Charakter der Einwohner“ Danzigs, dessen „erste Ingredienzien [...] Freiheitssinn und damit verbundene Vorliebe für die republikanische Regierungsform“ seien (ANONYM 1794:53). Dieser den Danzigern eigene Nationalcharakter habe bewirkt, dass sie sich Preußen so lange hartnäckig widersetzen konnten. Der Autor fügt jedoch einschränkend hinzu, dass die Danziger Bürger andererseits den „verderblichen Grundsätze[n] einer mißverstandenen Freiheit und Gleichheit“ gefolgt seien und unter dem „Schein von Freiheit“ in ihrer Stadt soziale Ungerechtigkeit geduldet hätten (ANONYM 1794:56). Im zehnten Brief stellt er fest:

Danzigs Staatsverfassung war gewiß sehr gut angelegt; die einzelnen Kräfte, welche zur Erhaltung des ganzen Staatskörpers wirken sollten, waren genau berechnet; der Staat war dazu klein, und jede Irregularität leicht bemerkbar; und doch artete auch hier die republikanische Regierungsform so leicht aus! Was will denn aus großen Republiken werden? (ANONYM 1794:80)

Der generell also positiv beurteilte Republikanismus sei in Danzig ,ausgeartet“, statt Gerechtigkeit habe Willkür geherrscht. Der letzte Satz zu den „großen Republiken“ bezeugt, dass der Verfasser sich mit diesen Überlegungen in Diskussionen über die republikanische Staatsform einschaltet, die im Umfeld der Französischen Revolution geführt wurden. Tatsächlich konnten in Danzig alle Bürger zumindest theoretisch an der politischen Ordnung partizipieren: Die Regierung (die sogenannte Erste Ordnung) bildeten vier Bürgermeister

IZABELLA GOLEC untersucht vor allem das Polenbild in den deutschen Reiseberichten, Samp hingegen bietet einen Überblick über das Bild Danzigs in der deutschen und polnischen Reiseliteratur von der ersten Teilung Polens bis zur Wiedererrichtung des polnischen Staates. 
(Proconsules) und der Städtische Rat, dem 23 Ratsherren (Consules) angehörten. Die Zweite Ordnung bestand aus dem Schöffengericht mit zwölf Mitgliedern und zur Dritten Ordnung gehörten 100 vom Rat ernannte Bürger. Dessen ungeachtet fühlten sich aber viele Kaufleute nicht durch den Rat, viele Handwerker nicht durch die Dritte Ordnung vertreten, ganz davon abgesehen, dass Angehörige unterer sozialer Schichten, etwa Hafenarbeiter, Bedienstete und Gesellen, gar kein Bürgerrecht erwerben konnten. Es kam daher in der Stadt immer wieder zu Protesten und Unruhen. ${ }^{2}$

Jerzy Samp attestiert den Briefen über Danzig einen „propreußischen, agitatorisch-propagandistischen Charakter“ (SAMP 1991:57). Nach meinem Eindruck geht das Anliegen des anonymen Verfassers jedoch über eine propreußische Propaganda hinaus. Wichtig ist ihm offenbar eine Kritik an der republikanischen Staatsform generell, an der die Danziger weiterhin festhalten wollten. Dass diese Kritik vor dem Horizont der französischen Revolution gelesen werden kann, legt auch eine vom Verfasser kolportierte Behauptung nahe, mit der Preußen die Besetzung Danzigs legitimierte, dass nämlich die Stadt einen gewissen „Monsieur Garnier“ aufgenommen habe, der „zu der französischen Propaganda gehörte“ und „von hier aus nach Berlin und anderen preußischen Gegenden [...] aufrührerische Briefe“ geschrieben haben soll (ANONYM 1794:57). ${ }^{3}$

Die Briefe über Danzig enthalten nicht nur Reflexionen zum Republikanismus, sondern auch Hinweise auf nötige Reformen und Ratschläge für die künftige Verwaltung der Stadt. Der Verfasser stellt sich zur Aufgabe, „denen, welche zu der neuen Staatsverfassung mitwirken sollen, nach meinen geringen Einsichten, einige Winke zu geben, wie und wo sie eigentlich verbessern können.“ (ANONYM 1794:Vorrede, o.S.) Er gibt den neuen Machthabern Empfehlungen, die sie beherzigen sollten, und benennt allerlei Missstände, die behoben werden müssten, etwa in sozialen Einrichtungen wie dem Lazarett, dem Waisenund Armenhaus, in Hospitälern, Gefängnissen und Schulen. Auch über die finanzielle Situation der Geistlichen, die Lage der Kaufleute und Gelehrten, die innere Ordnung und den Zustand der Straßen weiß er zu berichten. Während

2 Zur Regierungsform Danzigs und den entstandenen Konflikten und Unruhen vgl. LOEW (2011:114-117, 126 f.).

3 Der Historiker Gotthilf Löschin schreibt, die Stadt habe Joh. Joseph Garnier nach einem entsprechenden Untersuchungsverfahren an Berlin ausgeliefert, so dass der Vorwurf, sie habe ihn „beschützt“, nicht zutreffe (LÖSCHIN 1823:257). 
er Vorschläge zur Reformierung der einzelnen Institutionen unterbreitet, verleiht er immer wieder seiner Überzeugung Ausdruck, dass sich deren Lage unter der neuen preußischen Regierung verbessern werde.

Ein weiterer Text, der die Anfänge der preußischen Herrschaft über Danzig thematisiert, stammt aus der Feder von Carl B. Feyerabend, über den etwas mehr bekannt ist als über den Autor der Briefe über Danzig. Er wurde um 1765 in Danzig in einer mittellosen Familie geboren und arbeitete nach dem Studium in Königsberg bei einem russischen General in Livland zunächst als dessen Hauslehrer, dann als Adjutant. Gegen Ende des 18. Jahrhunderts war er als Lehrer an der Oberpfarrschule in St. Marien tätig. Er verfasste Kosmopolitische Briefe über die Geschichte des russischen Reiches (1803), eine Geschichte des polnischen Staates (1809) sowie Dramen und Schulbücher (GOLEC 1998:85). In den Jahren 1798 bis 1803 veröffentlichte er anonym in vier Bänden Kosmopolitische Wanderungen durch Preussen, Liefland, Kurland, Litthauen, Vollhynien, Podolien, Gallizien und Schlesien in den Jahren 1795-1797. In Briefen an einen Freund. Der Bericht über Danzig befindet sich im ersten Band „Germanien“.

In ihm schreibt Feyerabend von der „Wuth, die den Danziger befiel, sobald der Name Preuße genannt ward“ (ANONYM [FEYERABEND] 1798:98) und von dem „Schimmer der Hoffnung“, an den sich die Bevölkerung klammerte, als Tadeusz Kościuszko „zu kurz für Polens Glück, seine heldenvolle Rolle spielte, und die stolze Katharina zum erstenmale zitterte“ (ANONYM [FEYERABEND] 1798:113). Die Danziger Bürger hätten sich durch den polnischen Aufstand „schon im Geiste der Gewalt der Preußen entzogen“ gesehen (ANONYM [FEYERABEND] 1798:113). Ihr Hass auf die Preußen ist für Feyerabend durchaus verständlich, denn diese haben nicht nur nach der ersten Teilung Polens im Jahr 1772 Danzigs Handel schwer geschädigt, auch ihr Verhalten nach der Besetzung der Stadt sei eine „Schande“ gewesen: Sie „hauseten hier, wie einige ihrer Brüder am Rhein mit den Klubisten in Frankfurt und Mainz“ (ANONYM [FEYERABEND] 1798:106).

Feyerabend verurteilt die Teilungen Polens, für die in seinen Augen allerdings nicht Preußen, sondern Katharina II. verantwortlich ist; auch der polnische Adel und die Geistlichkeit hätten ihren Teil dazu beigetragen (vgl. GolEC 1998:86). Da er sich als einen Feind der Tyrannei bezeichnet, als jemanden, den „die gemißhandelten Rechte der Menschheit empören“ und der die Menschenrechte für „heilig und unabänderlich“ hält (ANONYM [FEYERABEND] 1798:48), verwundert es zunächst, dass auch er sich für die preußische Ord- 
nung ausspricht. Er lobt zwar Danzigs „,aristokratisch-republikanische Regierungsform“ (ANONYM [FEYERABEND] 1798:51), wenn er schreibt: „Die Konstituzion, auf welche die bisherige Regierungsform dieser Stadt gegründet war, hatte so wenig Tadelnswerthes an sich, daß sie vielmehr in vielen Stücken musterhaft genannt zu werden verdient hätte.“ Die Realisierung der Verfassung aber „ward oft Händen anvertraut, die sie mißbrauchten“ (ANONYM [FEYERABEND] 1798:54f.). Durch Willkür, unverhältnismäßige Steuern, Eigennutz und Korruption wuchsen Luxus auf der einen und soziales Elend auf der anderen Seite. Feyerabend hofft, dass die neue Regierung solche „sozialen Ungerechtigkeiten zu verhüten“ wisse (ANONYM [FEYERABEND] 1798:59), und glaubt, dass sich die Stände nun einander annähern würden, denn alle Bewohner der Stadt würden zu gleichberechtigten Staatsbürgern (ANONYM [FEYERABEND] 1798:154f.). Als Zusammenfassung seiner Ansicht kann folgendes Zitat gelten:

Der Danziger sprach immer mit Enthusiasmus von seinem unendlichen Republikanerglück, und zitterte vor der herannahenden Veränderung. Und als sich die Preußen vor den Thoren zeigten, und Einlaß begehrten, so schrie alles: ,Wir verlieren unsre Freiheit! Wir werden Sklaven! ‘ - Lieben Bürger, nehmt’s einem ehrlichen Mann nicht übel, der so redet, wie sein Herz denkt: Eure gerühmte Freiheit war meistentheils Traum, und Eure erwartete Sklaverei gab Euch Aussichten auf bessere Zeiten! (ANONYM [FEYERABEND] 1798:62)

August Leopold Graf von Lehndorff (1771-1820), Besitzer einiger Güter in West- und Ostpreußen und Autor des letzten Reiseberichts, auf den ich hier eingehe, äußert sich in seiner anonym herausgegebenen Reisebeschreibung Meine Reise ins blaue Ländchen, nebst Bemerkungen über Danzig. In Briefen an einen Freund (Danzig 1799) ähnlich kritisch wie der Verfasser der Briefe über Danzig und Carl B. Feyerabend über die Danziger Regierung. Die Freie Stadt sei eigentlich schon keine Republik mehr gewesen:

Wer die ehemalige Republik Danzig kannte, wird mir beipflichten, daß ihre gepriesene Freiheit und Unabhängigkeit schimärisch war, daß Despotie hier herrschte, und Parteilichkeit die Gerechtigkeit verdrängte. Danzig war ein Staat von geringem Umfange, jeder Fehler daher leicht zu übersehen, und dennoch verschwand allmählig die republikanische Regierungsform; Herrschaft und Reichthum kam in die Hände einzelner Familien, dadurch wurde der leidige Nepotismus erzeugt, und aus der Demokratie begann Aristokratie zu werden. (ANONYM [vON LEHNDORFF] 1799:139)

Die hier betrachteten Reiseberichte dokumentieren also die Ablehnung der preußischen Herrschaft durch eine Mehrheit der Danziger Bürger und verweisen darauf, dass diese eine negative Haltung zur politischen Verfasstheit des 
preußischen Staates einschloss. Die Danziger schmerzte es nicht nur, ihre politische und wirtschaftliche Selbstständigkeit aufgeben zu müssen. Auch der Abschied von ihrer republikanischen Verfassung fiel ihnen schwer. Die hier zitierten Autoren hielten den aufgeklärten Absolutismus Preußens im Vergleich zum Republikanismus allerdings für den besseren Weg, soziale Gerechtigkeit und Gleichheit vor dem Gesetz zu garantieren. Es ist nicht auszuschließen, dass sie die neue Herrschaft auf diese Weise legitimieren wollten, zumal sie alle in Preußen lebten und ihr Wirken mit diesem Staat verbanden. Andererseits betteten sie ihre Kommentare in eine Diskussion über den Republikanismus und über die ideale Staatsform ein und verurteilten keineswegs die Demokratie an sich, meinten aber, diese sei in Danzig missbraucht worden.

\section{Johannes Daniel Falk: Leben, wunderbare Reisen und Irrfahrten des Johannes von der Ostsee (1805)}

Johannes Daniel Falk, 1768 in Danzig als Sohn eines Handwerkers geboren, konnte dank Stipendien des Danziger Senats das Akademische Gymnasium besuchen und ein Theologiestudium an der Fridericiana in Halle aufnehmen, das er allerdings bald abbrach, da er sich der Literatur stärker verbunden fühlte. Von 1797 bis zu seinem Tode im Jahr 1826 lebte er in Weimar; nach Danzig kehrte er seit seiner Schulzeit nicht wieder zurück.

Falk ist heute vor allem als Begründer der Sozialpädagogik und Verfasser des Weihnachtsliedes $O$ du fröhliche ... bekannt, unter seinen Zeitgenossen machte er sich aber zuerst als Satiriker einen Namen. Nach Ansicht von Richard John Allen war er der letzte bedeutende Vertreter dieser literarischen Tradition in Deutschland. Bis heute werde sein Werk unterschätzt (vgl. DEMANDT 1999:13). Eines der zu wenig beachteten Werke Falks ist gewiss der autobiographische Roman Leben, wunderbare Reisen und Irrfahrten des Johannes von der Ostsee aus dem Jahr $1805,{ }^{4}$ der von einer Kindheit und Jugend im Danzig der 1770er bis 1790er Jahre erzählt. Der Fokus dieses Werkes, einer Verschränkung von Entwicklungs- und Briefroman, liegt auf den Bildungserlebnissen des jungen Johannes, der aus einer armen Handwerkerfamilie stammt

4 Er wurde seit 1805 nicht wieder aufgelegt, nur Auszüge wurden neu veröffentlicht, so in FALK 1886 und 1922. In der Encyklopedia Gdańska, einem Grundlagenwerk zur Danziger Geschichte, wird der Roman nicht erwähnt, vgl. KOTARSKI 2012. 
und sich in ein Mädchen aus einem der reichsten Patrizierhäuser der Stadt verliebt. ${ }^{5}$ Die Beziehung zwischen ihnen leidet nicht nur unter dem sozialen Unterschied, sondern auch unter Missverständnissen, die aus gegenseitigem Misstrauen entstehen. Die Handlung des Romans wird in mehreren Briefwechseln erzählt. Johannes und seine geliebte Jeanette schreiben sich Briefe, letztere zieht ihre Cousine schriftlich ins Vertrauen und Johannes korrespondiert zusätzlich noch mit seinem „Vetter aus Preußen“ (FALK 1805:38).

Die drei Korrespondenzen verleihen dem Roman nicht nur Polyphonie und eine mehrperspektivische Narration, sondern auch eine dem Drama ähnelnde Struktur der Rede und Widerrede, die es u.a. erlaubt, gegensätzliche Meinungen zur preußischen Besetzung Danzigs zu präsentieren. Auch zwei gegenläufige Handlungssequenzen stellen in Korrelation zur Raumsignatur des Textes zwei Haltungen zu diesem historischen Ereignis einander gegenüber. Zwischen dem Vetter, der nach der ersten Teilung Polens als Hauptmann der preußischen Armee bereits in der Nähe von Danzig stationiert ist, und Jeanettes Cousine entsteht nämlich eine weitere Liebesbeziehung, und der Preuße lässt sich nach der Einnahme der Stadt und der Heirat mit der Danzigerin in deren Heimatstadt nieder. Von dort aus lädt er Johannes, der inzwischen in Wien lebt, zur Rückkehr ein, allerdings müsse dieser nun „nach unserer [der preußischen, M.B.] Pfeife tanzen“ (FALK 1805:239). Obwohl Johannes und Jeanette das sie trennende Missverständnis am Ende aufklären können und ebenfalls zu heiraten beabsichtigen, kommt für den Protagonisten eine Rückkehr ins nun preußische Danzig nicht in Frage.

Der historische Hintergrund der Bildungs- und Liebesgeschichte, also die preußische Herrschaft im Danziger Umland (seit 1772) und die Einnahme der Stadt durch die preußische Armee, wird somit in die Romanhandlung direkt integriert. Von beidem wird aus der Perspektive mehrerer Figuren erzählt, die

Demandt weist auf die Parallelen zwischen der Bildungs- und Liebesgeschichte des Romans und der Biographie von Falk hin (vgl. DEMANDT 1999:14 sowie seine Ausführungen zu den einzelnen Ereignissen). Handschriftliche Anmerkungen in einem Exemplar des Romans in der Danziger Bibliothek der Akademie der Wissenschaften legen zudem nahe, dass Falk originale Brief- und Tagebuchauszüge in den Text direkt integriert hat. So findet sich neben dem letzten Brief im Roman die Notiz „Weimar 1805. Aus meinem Tagebuch“ und neben dem „Sechste[n] Brief von Johannes von der Ostsee an seinen Vetter in Preußen“ (FALK 1805:177) ein Vermerk zum Entstehungsort Halle. Falk könnte diese Erläuterungen für einen Freund in Danzig, dem er ein Exemplar des Buches zukommen ließ, hinzugefügt haben. 
auch verschiedene Haltungen gegenüber den neuen Herrschern in Danzig präsentieren. Während Johannes das Ende der Freien Stadt als ein Unglück empfindet, das er nur durch künstlerische Tätigkeit mental überwinden kann, hat Jeanettes Cousine nach eigenen Worten „den Feind“ schon lange vor der Besetzung der Stadt ,in meinem Herzen einquartiret“ (FALK 1805:175). Ihr mit der Einnahme Danzigs zum Major avancierter Ehemann berichtet wiederum davon, dass die Danziger Bürger, als sie zum Lob der russischen Siege während des Kościuszko-Aufstandes von 1794 in den Kirchen ein „Te Deum“ anstimmen sollten, laut ein „Thad däum laudamus“ sangen, um so ihre Sympathie für Tadeusz Kościuszko zu bekunden (FALK 1805:238).

In dem Briefwechsel, den Johannes mit seinem Vetter führt, wird die Preußenkritik unmittelbar formuliert. ${ }^{6}$ Während der Hauptmann die preußische Politik gegenüber Polen und der Freien Stadt legitimiert, versucht Johannes ihn davon zu überzeugen, dass sowohl Danzig als auch die Polen im Danziger Umland gut ohne den Fortschritt leben könnten, den ihnen Preußen aufzwinge. Als Kernstück der Polemik zwischen dem preußischen und dem Danziger Briefschreiber ist in ihre Korrespondenz eine Satire auf Fichtes Schrift Der geschloßne Handelsstaat. Ein philosophischer Entwurf als Anhang zur Rechtslehre und Probe einer künftig zu liefernden Politik (1800) eingefügt. Falk legt sie einer historisch realen Person in den Mund, nämlich Samuel Ludwig Majewski, dem ersten Pastor an der St. Petri-Kirche, der in der Danziger Zeit sein wichtigster Förderer und Lehrer war. ${ }^{7}$ Er sei - so Johannes, so aber auch

$6 \quad$ Sie äußerte sich bereits in einem früheren Werk, dem Puppenspiel Die Uhu '. Eine dramatisch-satirische Rhapsodie mit Chören von Uhu'n, Raben und Nachteulen von 1796, vgl. DEMANDT 1999:208f., HEUFERT 2008:47-50.

7 Samuel Ludwig Majewski wurde als Sohn eines polnischen Predigers 1736 in Lissa geboren und starb 1801 in Danzig. Nach dem Studium in Königsberg und Utrecht sowie Reisen nach England, Frankreich und in die Schweiz war er als evangelischer Geistlicher in Danzig und Petersburg tätig. Zu seinen Publikationen gehören neben religiösen Schriften wie die Psalmen und Lieder zum Gebrauch der Evangelischreformirten Gemeine in Danzig (1785) auch Gelegenheitsreden, die 1775 bei Karl Benjamin Lengnich in Leipzig erschienen. Zur Bedeutung Majewskis als Förderer und Lehrer von Falk siehe DemandT 1999:47-99. Den Aufzeichnungen Falks über die Gespräche mit Majewski lässt sich entnehmen, dass dieser ein entschiedener Kritiker Friedrichs des Großen war, und Demandt vermutet, dass Falks Freiheitsdenken und Danziger Patriotismus nicht nur durch das „hugenottische Erbe“ seiner Eltern, sondern auch durch seinen Lehrer Majewski beeinflusst wurde (DEMANDT 1999:49f.). 
Falk an anderer Stelle über ihn ${ }^{8}$ - ein „großer Patriot und ein abgesagter Feind von jeder Verfassung, welche die Menschen zu Maschinen macht.“ (FALK 1805:87) Schon diese Charakterisierung lässt eine Ablehnung des preußischen Staates vermuten. Der Aufsatz mit dem Titel Etwas über die freyen deutschen Reichsstädte, in Bezug auf Fichtens geschlossenen Handelsstaat (FALK 1805:88) setzt zu Beginn ein Gleichheitszeichen zwischen geistiger Freiheit und dem Handel freier Städte: „Man kann [...] kek und zuversichtlich behaupten: daß, so lange noch der Handel in der City zu London blüht, auch der freye Geist dieser stolzen Insulaner nie aussterben wird.“ (FALK 1805:88). Der von Fichte entworfene „geschlossene Handelsstaat“ ersticke hingegen jegliche selbstständige Regung seiner Bürger. Er nehme ihnen alles Eigentum und sorge dafür

im Überfluß für Essen, Trinken, Kleider, Schuh, und alles Übrige. Dem zu Folge stellt er die genausten Berechnungen an, und damit der Staat nicht etwa mehr Lichter gießt, als er bedarf, oder mehr Brillen schleift, als er gerade für die Nasen, die man ihm dreht, nöthig hat; so werden ordentliche Cantons eingerichtet, worin die Lichtgießer, Brillenschleifer, Schuster, Schneider, Beker, Fleischer, Schmiede, förmlich wie die Soldaten, enrolirt werden. Auch die Dichter und Künstler kommen daran. Wenn demnach ein junger Dichter, wie z. B. Klopstock, oder ein junger Mahler, wie z. B. Raphael, Lust hat, in den Mahler- oder DichterCanton aufgenommen zu werden: so hat er sich zuförderst bey dem Herrn CantonInspector zu melden. Die Canton-Register werden darauf nachgeschlagen, und wenn sich zu allem Malheur findet, daß keine Stellen vacant, oder daß Dichter und Mahler eben complet sind: so muß Supplikant nolens volens in den sauern Apfel beißen, und ein ander Handwerk lernen. Klopstock kann etwa statt seiner Messiade - Handschuh machen; Raphael statt der Zeichnungen zu seinen Tapeten, Potsdammer Zopfband verfertigen: und beide werden schon, wenn sie gute Patrioten sind, darüber zu trösten seyn. (FALK 1805:90f.)

Der ,Versorgungsstaat‘ plant nicht nur, was seine Bürger benötigen, produziert nicht nur die entsprechenden Waren für sie, sondern er regelt durch seine Bürokratie auch Bildung und Kunst. Da er als „geschlossener Handelsstaat“ keinen Außenhandel betreibt, muss er alles, was er benötigt, selbst herstellen bzw. die Rohstoffe, die ihm aufgrund seiner geographischen Lage fehlen, durch andere substituieren: So können den Zucker etwa Runkelrüben ersetzen, den Kaffee Zichorien und statt Zobelpelze können die Frauen Pelze von Bären tragen. Wenn der Staat aber für bestimmte Rohstoffe keinen Ersatz findet, ergreift

Im handschriftlichen Bücherkatalogus von 1805, Goethe-Museum Düsseldorf, Kippenberg-Stiftung, Falk II.11, 6f., Angabe nach DEMANDT 1999:51. 
er Besitz von einem Land, das über sie verfügt. Die Annexion geschieht auf ganz ,friedliche“ Weise:

Sollte indeß der Abgang einiger Artikel, wie z. B. der des Wein's für gewiße Individuen, besonders für die, welche eine sitzende Lebensart führen, gar zu genant und drükend seyn: so liegt die Schuld bloß daran, daß der Vernunftstaat noch nicht hinlänglich geschlossen ist. Herr Fichte legt es ihm daher als Pflicht auf, ,in seine natürliche Grenzen einzurüken', d.h. in vorstehendem Fall von den Weinländern ohne weiteres Besitz zu nehmen. Denn da er so immer unter allen seinen Nachbarn den größten Schaz, folglich auch die größte Armee, auf den Beinen hat: so wird dies Besitznehmen, wie Hr. Fichte meint, ohne großes Blutvergießen zu Stande kommen, und sich, mit einer bloßen Demonstration, von Seiten des Stärkeren gegen die Schwächern, bald machen lassen. (FALK 1805:91f.)

In seiner Satire auf den preußischen Staat wirft Falk also ebenso wie der anonyme Autor der Briefe über Danzig, Carl B. Feyerabend und August Graf von Lehndorff anlässlich des Untergangs der Freien Stadt Danzig die Frage nach der idealen Staatsform auf. Doch seine Antwort fällt anders aus als die der angeführten Reiseschriftsteller. Während jene der Demokratie den aufgeklärten Absolutismus des preußischen Staates vorziehen, verteidigt Falk den Republikanismus gegenüber der preußischen Ordnung, die in seinen Augen den Bürgern Unfreiheit und den Nachbarn Feindseligkeit, ja Krieg bringt. Dem alles regulierenden „Vernunftstaat“ zieht er das Leben in einer „freyen deutschen Reichs[stadt]“ (FALK 1805:88) vor, wo mit dem internationalen Handel auch Weltoffenheit und geistige Freiheit existieren (siehe auch FALK 1805:82f.). Den im Roman Leben, wunderbare Reisen und Irrfahrten des Johannes von der Ostsee angeschlagenen Ton wird gut dreißig Jahre später Johanna Schopenhauer aufgreifen, wenn sie in ihren Jugenderinnerungen die kulturelle und religiöse Vielfalt Danzigs preist, ihre Abneigung gegenüber Preußen herausstellt und sich voller Stolz eine „freie Republikanerin“ (SCHOPENHAUER 2000:199) nennt.

\section{Literatur}

Anonym (1794): Briefe über Danzig. Berlin.

ANONYM [FEYERABEND, CARL B.] (1798): Kosmopolitischen Wanderungen durch Preussen, Liefland, Kurland, Litthauen, Vollhynien, Podolien, Gallizien und Schlesien in den Jahren 1795-1797. In Briefen an einen Freund. Danzig.

ANONYM [VON LEHNDORfF, August LeOPOLd] (1799): Meine Reise ins blaue Ländchen, nebst Bemerkungen über Danzig. In Briefen an einen Freund. Danzig. 
DEMANDT, Johannes (1999): Johannes Daniel Falk. Sein Weg von Danzig über Halle nach Weimar (1768-1799) (= Arbeiten zur Geschichte des Pietismus 36). Göttingen.

FALK, Johannes DANIEL (1805): Leben, wunderbare Reisen und Irrfahrten des Johannes von der Ostsee. Tübingen.

Falk, Johannes Daniel (1886): Die Besitznahme Danzigs durch die Preußen am 4. April 1793: nach einem höchst seltenen Briefwechsel Johannes Daniel Falk‘s. Hrsg. und kommentiert von Emil König. Rathenow.

FALK, Johannes Daniel (1922): Jugendgeschichte des Johannes von der Ostsee u. a. Geschichten. Hrsg. von Ludwig Mahlau. Danzig.

Golec, Izabella (1998): Danzig zwischen der I. und III. Teilung in der deutschen Reiseliteratur. In: 1000 Jahre Danzig in der deutschen Literatur. Studien und Beiträge (= Studia Germanica Gedanensia 5). Hrsg. v. Marek Jaroszewski. Gdańsk, 77-91.

Heufert, GeRHARD (2008): Johannes Daniel Falk. Satiriker, Diplomat und Sozialpädagoge. Weimar.

JÄGER, HANS-Wolf (1998): Danzig in der deutschen Reiseliteratur des 18. und frühen 19. Jahrhunderts. In: 1000 Jahre Danzig in der deutschen Literatur. Studien und Beiträge (= Studia Germanica Gedanensia 5). Hrsg. v. Marek Jaroszewski. Gdańsk, 61-75. Loew, Peter Oliver (2011): Danzig. Biographie einer Stadt. München.

Löschin, GotTHILF (1823): Geschichte Danzigs von der ältesten bis zur neuesten Zeit. Mit beständiger Rücksicht auf Cultur der Sitten, Wissenschaften, Künste, Gewerbe und Handelszweige. Bd. 2., 2. überarb. Auflage. Danzig.

SAMP, JERZY (1991): Gdańsk w relacjach z podróży 1772-1918 (= Zeszyty naukowe Uniwersytetu Gdańskiego. Rozprawy i monografie 152). Gdańsk.

SCHOPENHAUER, JohANNA (2000): Im Wechsel der Zeiten, im Gedränge der Welt. Jugenderinnerungen, Tagebücher, Briefe. Düsseldorf, Zürich. 